\title{
6

\section{Treatment Using the SpyGlass Digital System in a Patient with Hepatolithiasis after a Whipple Procedure}

\author{
Hirofumi Harima', Kouichi Hamabe', Fusako Hisano ${ }^{1}$, Yuko Matsuzaki', Tadahiko Itoh², Kazutoshi Sanuki and Isao Sakaida ${ }^{3}$ \\ Department of ${ }^{1}$ Gastroenterology, ${ }^{2}$ Cancer Screening, Ube Industries Central Hospital, Ube, Yamaguchi, ${ }^{3}$ Department of Gastroenterology and \\ Hepatology, Yamaguchi University Graduate School of Medicine, Ube, Yamaguchi, Japan
}

An 89-year-old man was referred to our hospital for treatment of hepatolithiasis causing recurrent cholangitis. He had undergone a prior Whipple procedure. Computed tomography demonstrated left-sided hepatolithiasis. First, we conducted peroral direct cholangioscopy (PDCS) using an ultraslim endoscope. Although PDCS was successfully conducted, it was unsuccessful in removing all the stones. The stones located in the B2 segment were difficult to remove because the endoscope could not be inserted deeply into this segment due to the small size of the intrahepatic bile duct. Next, we substituted the endoscope with an upper gastrointestinal endoscope. After positioning the endoscope, the SpyGlass digital system (SPY-DS) was successfully inserted deep into the B2 segment. Upon visualizing the residual stones, we conducted SPY-DS-guided electrohydraulic lithotripsy. The stones were disintegrated and completely removed. In cases of PDCS failure, a treatment strategy using the SPY-DS can be considered for patients with hepatolithiasis after a Whipple procedure.

Clin Endosc 2018;51:596-599

Key Words: Hepatolithiasis; Whipple procedure; Cholangioscopy; SpyGlass digital system

\section{INTRODUCTION}

Hepatolithiasis is a postoperative complication of pancreatoduodenectomy. Stone removal under direct visualization has clinical benefits compared with the fluoroscopy method because complete stone clearance is important for preventing recurrent stones and/or cholangitis after treatment. ${ }^{1}$ The efficacy of peroral cholangioscopy (POCS) for the treatment of hepatolithiasis has been previously demonstrated in patients with normal gastrointestinal (GI) anatomy; ${ }^{2}$ however, POCS has rarely been reported in patients with altered GI anatomy. Applying POCS in patients with altered GI anatomy is generally difficult due to the long distance necessary for passage. In such

Received: March 14, 2018 Revised: April 12, 2018

Accepted: April 13, 2018

Correspondence: Hirofumi Harima

Department of Gastroenterology, Ube Industries Central Hospital, 750 Nishikiwa, Ube, Yamaguchi 755-0042, Japan

Tel: +81-836-519968, Fax: +81-836-519454, E-mail: harima@yamaguchi-u.ac.jp ORCID: https://orcid.org/0000-0003-0406-0345

(cc) This is an Open Access article distributed under the terms of the Creative Commons Attribution Non-Commercial License (http://creativecommons.org/ licenses/by-nc/3.0) which permits unrestricted non-commercial use, distribution, and reproduction in any medium, provided the original work is properly cited. patients, the effectiveness of peroral direct cholangioscopy (PDCS) using an ultraslim endoscope has been reported. ${ }^{3}$ However, the PDCS success rate cannot reach $100 \%$ because in some cases, the intrahepatic bile duct (IHBD) is too small for the insertion of an ultraslim endoscope, thereby preventing PDCS from being conducted. ${ }^{4}$ In the current report, we describe the case of a patient with hepatolithiasis after a Whipple procedure who was successfully treated with POCS using a new digital cholangioscope, the SpyGlass digital system (SPY-DS, outer diameter 3.5 mm; Boston Scientific Corp., Natick, MA, USA).

\section{CASE REPORT}

An 89-year-old man was referred to our hospital for treatment of hepatolithiasis causing recurrent cholangitis. He had undergone a prior Whipple procedure due to an ampullary carcinoma. Computed tomography demonstrated left-sided hepatolithiasis (Fig. 1). First, we conducted PDCS with endoscopic retrograde cholangiography using an ultraslim endoscope (EG530NW, outer diameter $5.9 \mathrm{~mm}$; Fujifilm, Tokyo, Japan), which showed large stones in the common trunk of the IHBD of seg- 
ment 2 (B2) and segment 3 (B3) (Fig. 2). Although PDCS was performed successfully in terms of viewing and reducing the volume of stones (Fig. 3), it was unsuccessful in removing all the stones. The stones located in the B2 segment were difficult to remove because the endoscope and the cholangiography catheter could not be inserted deeply into this segment due to the small size and curved shape of the IHBD. Next, we substituted the endoscope with an upper GI endoscope that had a large working channel of 3.7 mm (GIF-1T240; Olympus Med- ical Systems, Tokyo, Japan). After positioning the endoscope in the bilioenteric anastomosis, the SPY-DS was successfully inserted deeply into the $\mathrm{B} 2$ segment. Upon visualizing the residual stones, we conducted SPY-DS-guided electrohydraulic lithotripsy (EHL, AUTOLITH; Nortech, Elgin, IL, USA). The stones were subjected to disintegration combined with frequent irrigation and were removed completely (Video 1). No procedure-related complications occurred.
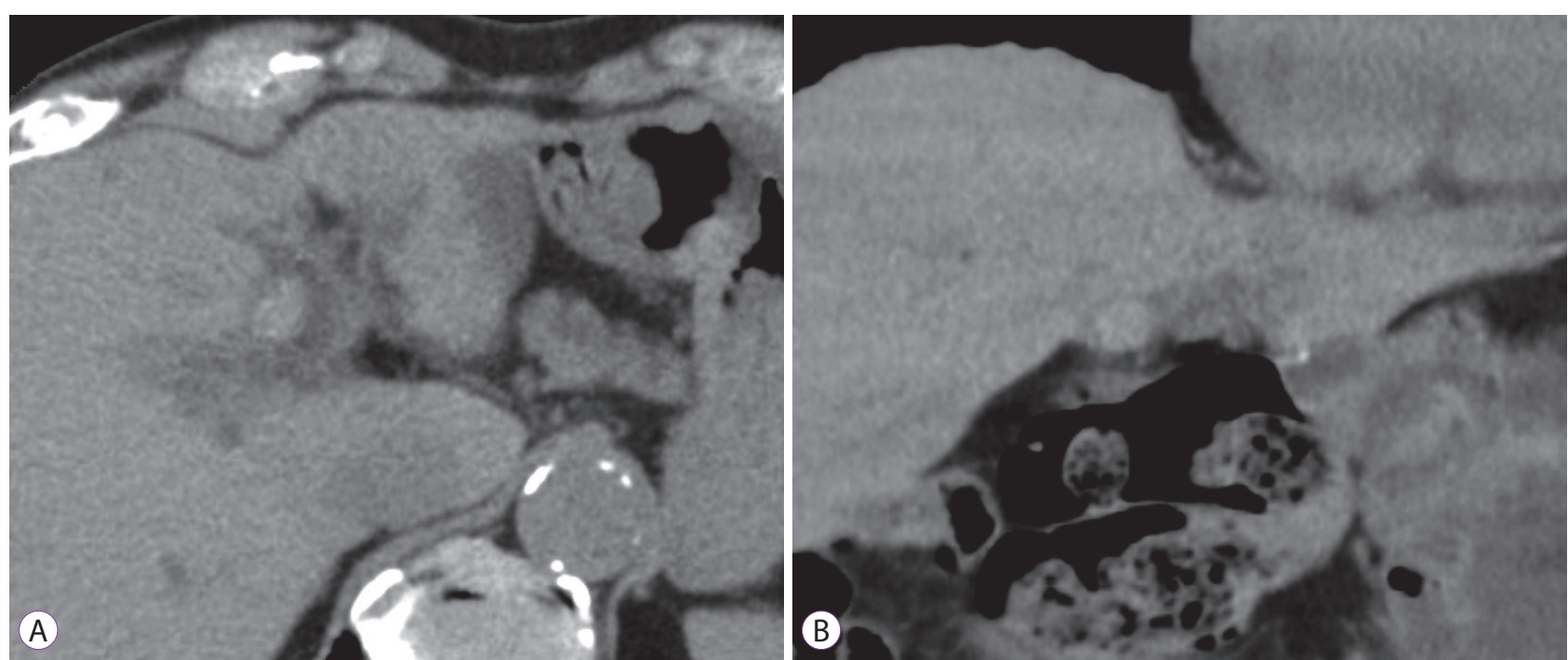

Fig. 1. Computed tomography results. (A) Axial view. (B) Coronal view. Computed tomography images showing a calcified stone impacted within the intrahepatic bile duct of the left lobe.
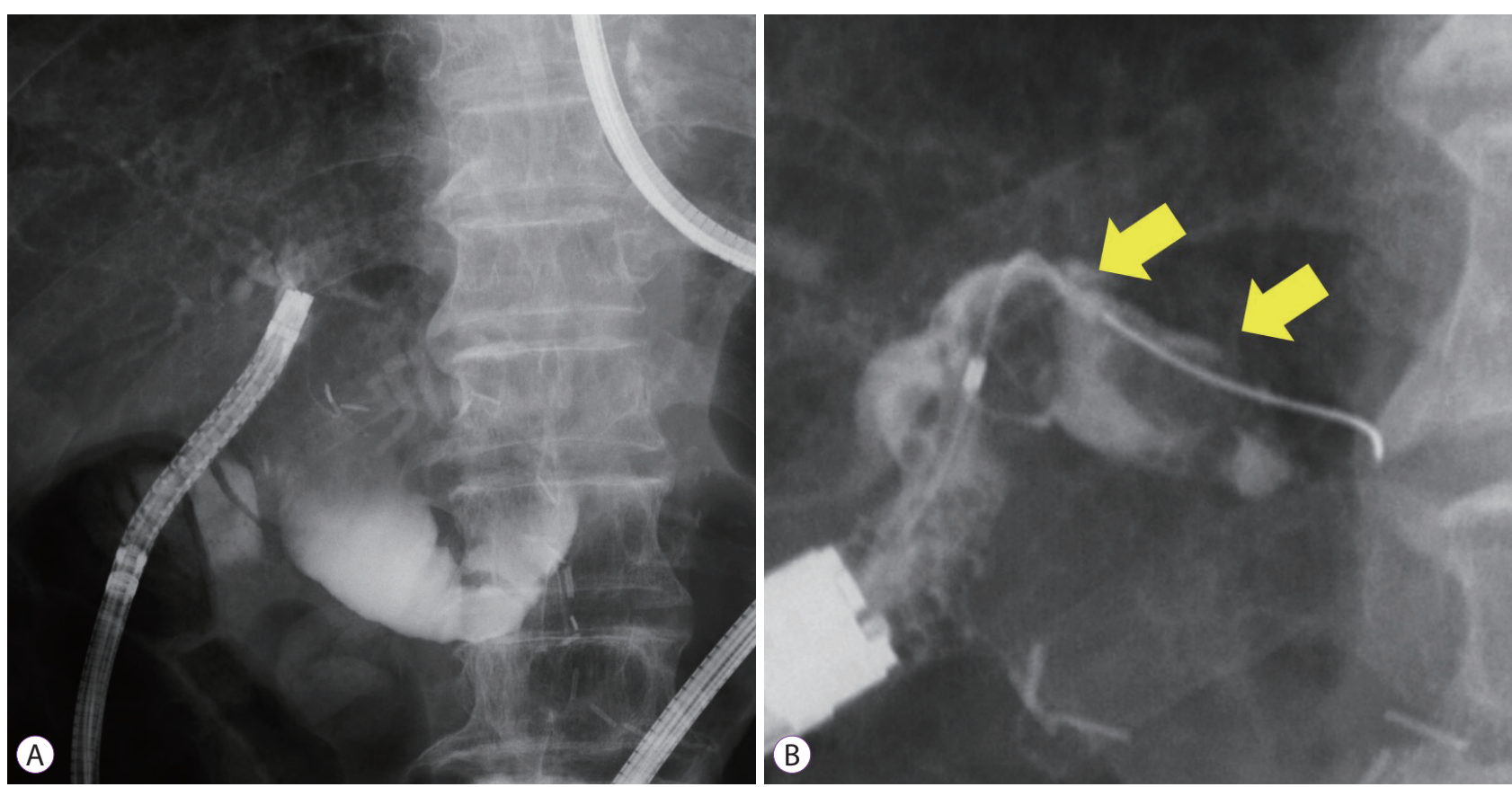

Fig. 2. Cholangiography results. (A) An ultraslim endoscope was inserted directly into the left hepatic duct. (B) Cholangiography image showing two large stones in the common trunk of the intrahepatic bile duct of segment 2 and segment 3 (arrows). 

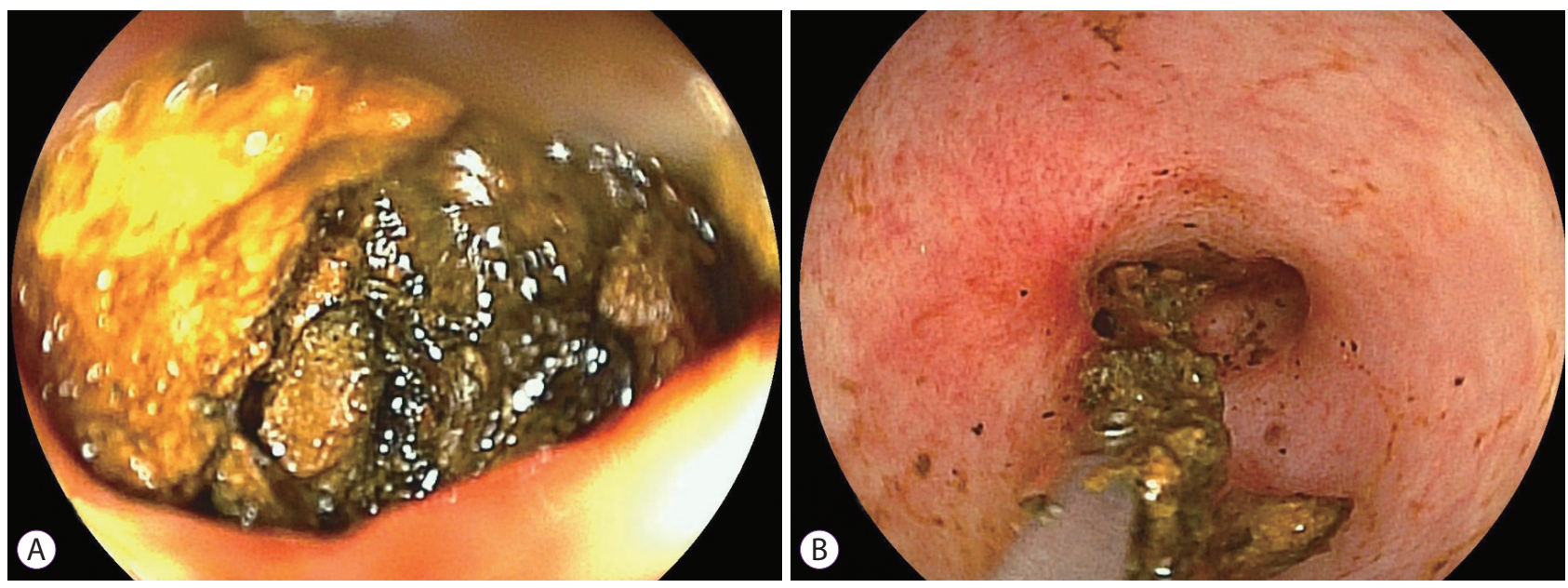

Fig. 3. Peroral direct cholangioscopy (PDCS) results. (A) PDCS image showing a large stone located in the common trunk of the intrahepatic bile duct of segment 2 and segment 3. (B) The volume of the stone was reduced using biopsy forceps during PDCS.

\section{DISCUSSION}

Since the 1970s, POCS has been used in the diagnosis and treatment of a variety of biliary diseases. ${ }^{5}$ The first POCS procedure, known as "mother-baby" cholangioscopy, used a very slim video cholangioscope (CHF-B260; Olympus Medical Systems) that was introduced through the working channel of a duodenoscope. Although this system yields good quality images, several limitations prohibit its widespread use, including the need for two endoscopists, the cost, and scope fragility. ${ }^{6} \mathrm{~A}$ single-operator fiberoptic cholangioscope system, the SpyGlass direct visualization system (Boston Scientific Corp.), has been available since $2005{ }^{7}$ However, although the cholangioscope was improved via 4-way deflected steering by a single operator, ${ }^{8}$ the image quality was poor. In 2014, the second generation of the cholangioscope, the SPY-DS, was introduced, with higher quality images, improvements in ergonomics, stability, and accessory exposure, and a larger working channel. ${ }^{9}$ The clinical utility of the SPY-DS for diagnostic and therapeutic procedures in biliary disease has been reported in patients with normal GI anatomy ${ }^{10}$ but has rarely been reported in patients with altered GI anatomy. To use the SPY-DS, an endoscope with a working channel larger than $3.7 \mathrm{~mm}$ is required. Although the effectiveness of short, double-balloon and single-balloon enteroscopes for treating biliary disease in patients with altered GI anatomy has been reported, ${ }^{11,12}$ the SPY-DS cannot be inserted through such enteroscopes because they have a working channel smaller than $3.2 \mathrm{~mm}$. A PDCS trial using an ultraslim endoscope to treat biliary disease under visualization in patients with altered GI anatomy has been reported. ${ }^{3}$ In the present case, although we also attempted PDCS using an ultraslim endoscope, we failed to achieve complete stone removal because the diameter of the IHBD was smaller than that of the ultraslim endoscope
(5.9 mm). A previous study revealed that the PDCS procedure using an ultraslim endoscope failed in 14\% (2/14) of patients due to the large diameter of the scope. ${ }^{4}$ While the results are dependent on the particular case, an upper GI endoscope is often able to reach the bilioenteric anastomosis in patients after a Whipple procedure. When an endoscope with a large-diameter working channel can reach the bilioenteric anastomosis, POCS using the SPY-DS may be considered. In the present case, POCS was successfully conducted using the SPY-DS because the diameter of the IHBD was larger than that of the SPY-DS $(3.5 \mathrm{~mm})$. Once the SPY-DS is inserted into the IHBD, the approach for a targeted biliary duct is simplified due to the use of 4-way deflected steering and good visibility. After the stones are visualized, they can be disintegrated and removed using EHL or a laser lithotripter. ${ }^{13}$

Recently, endoscopic ultrasound-guided biliary drainage has been developed, and an antegrade stone removal technique using the SPY-DS through a fistula between the IHBD and the stomach or intestine has been reported..$^{14}$ However, endoscopic, ultrasound-guided biliary drainage can cause severe adverse events, such as perforation or peritonitis, ${ }^{15}$ and its use may be limited according to the operator's expertise. When the bilioenteric anastomosis is accessible after GI reconstruction, the endoscopic biliary approach via the intestinal tract is believed to be safer than the endoscopic ultrasound-guided biliary approach.

In conclusion, POCS using the SPY-DS contributed to complete stone removal in a patient with hepatolithiasis after a Whipple procedure. In cases of PDCS failure, a treatment strategy using the SPY-DS may be considered.

\section{Conflicts of Interest}

The authors have no financial conflicts of interest. 


\section{Supplementary Material}

Video 1. Use of SpyGlass digital system-guided electrohydraulic lithotripsy to disintegrate and remove stones located deep in the bile duct of segment 2 (B2). (https://doi.org/10.5946/ce.2018.057.v001).

\section{REFERENCES}

1. Cheon YK, Cho YD, Moon JH, Lee JS, Shim CS. Evaluation of long-term results and recurrent factors after operative and nonoperative treatment for hepatolithiasis. Surgery 2009;146:843-853.

2. Okugawa T, Tsuyuguchi T, K C S, et al. Peroral cholangioscopic treatment of hepatolithiasis: long-term results. Gastrointest Endosc 2002;56:366371.

3. Itoi T, Sofuni A, Itokawa F, et al. Diagnostic and therapeutic peroral direct cholangioscopy in patients with altered GI anatomy (with videos). Gastrointest Endosc 2012;75:441-449.

4. Matsumoto K, Tsutsumi K, Kato H, et al. Effectiveness of peroral direct cholangioscopy using an ultraslim endoscope for the treatment of hepatolithiasis in patients with hepaticojejunostomy (with video). Surg Endosc 2016;30:1249-1254.

5. Ghersi S, Fuccio L, Bassi M, Fabbri C, Cennamo V. Current status of peroral cholangioscopy in biliary tract diseases. World J Gastrointest Endosc 2015;7:510-517.

6. Xu MM, Kahaleh M. Recent developments in choledochoscopy: technical and clinical advances. Clin Exp Gastroenterol 2016;9:119-124.

7. Parsi MA. Peroral cholangioscopy in the new millennium. World J Gas- troenterol 2011;17:1-6.

8. Chen YK, Pleskow DK. SpyGlass single-operator peroral cholangiopancreatoscopy system for the diagnosis and therapy of bile-duct disorders: a clinical feasibility study (with video). Gastrointest Endosc 2007;65:832841.

9. Franzini TA, Moura RN, de Moura EG. Advances in therapeutic cholangioscopy. Gastroenterol Res Pract 2016;2016:5249152.

10. Ogura T, Imanishi M, Kurisu Y, et al. Prospective evaluation of digital single-operator cholangioscope for diagnostic and therapeutic procedures (with videos). Dig Endosc 2017;29:782-789.

11. Shimatani M, Matsushita M, Takaoka M, et al. Effective "short" double-balloon enteroscope for diagnostic and therapeutic ERCP in patients with altered gastrointestinal anatomy: a large case series. Endoscopy 2009;41:849-854.

12. Iwai T, Kida M, Yamauchi H, Imaizumi H, Koizumi W. Short-type and conventional single-balloon enteroscopes for endoscopic retrograde cholangiopancreatography in patients with surgically altered anatomy: single-center experience. Dig Endosc 2014;26 Suppl 2:156-163.

13. Trikudanathan G, Navaneethan U, Parsi MA. Endoscopic management of difficult common bile duct stones. World J Gastroenterol 2013;19:165173.

14. Kamiyama R, Ogura T, Okuda A, et al. Electrohydraulic lithotripsy for difficult bile duct stones under endoscopic retrograde cholangiopancreatography and peroral transluminal cholangioscopy guidance. Gut Liver 2018 Feb 8 [Epub]. https://doi.org/10.5009/gnl17352.

15. Iwashita T, Doi S, Yasuda I. Endoscopic ultrasound-guided biliary drainage: a review. Clin J Gastroenterol 2014;7:94-102. 